\title{
Limited capacity information processing and pigeon matching-to-sample: Testing alternative hypotheses
}

\author{
CYNTHIA M. LANGLEY and DONALD A. RILEY \\ University of California, Berkeley, California
}

\begin{abstract}
The limited capacity hypothesis explains the element superiority effect observed in pigeons' element-compound matching-to-sample performance as the result of a central information processing overload occurring at the time of sample exposure. Major alternative hypotheses offered in the literature to date argue that element superiority is due to a difference in element- and compound-sample memory codes or to a peripheral sensory limitation during sample exposure. These alternative factors were simultaneously prevented from influencing matching performance in the present experiment, but the element superiority effect remained. A central information processing account of the element superiority effect is supported by the strong tests of alternative hypotheses provided herein. The discussion addresses remaining challenges to the hypothesis that information overload for compound samples occurs at the time of sample exposure.
\end{abstract}

In an extensive literature on the results of elementcompound delayed matching-to-sample (DMTS) with pigeons, matching performance following a color- or lineelement sample is superior to performance following a compound sample consisting of both color and line elements (Cook 1980; Cook, Riley, \& Brown, 1992; Lamb, 1988; Maki \& Leith, 1973; Maki \& Leuin, 1972; Maki, Riley, \& Leith, 1976; Roberts \& Grant, 1978; Santi, Grossi, \& Gibson, 1982). One interpretation, the limited capacity hypothesis, asserts that the element superiority effect is attributable to information overload during initial encoding of the compound while it is physically present. Three major alternative hypotheses have been offered in the literature as accounts of element superiority: generalization decrement, coding decrement, and gaze direction. Until now, the experimental approach for pitting the limited capacity hypothesis against these alternatives has been to create experimental designs in which one alternative factor is prevented from affecting matching performance and to conclude, if element superiority is still found, that the controlled factor is not important in producing element superiority. Hindsight suggests that this approach is not adequate. All three of the alternative factors may contribute to some degree to element superiority, and the removal of any one factor might be expected to have relatively little effect on performance. The current experiment simultaneously controls for all three of the major alternative hypotheses offered in the literature to

This research was supported by NIMH Grant MH44746 to D.A.R. We thank Geoffrey Keppel for his assistance with statistical analyses. Thanks also to Marvin Lamb, Anthony Wright, and an unidentified reviewer for helpful comments on previous versions of the manuscript. Requests for reprints should be sent to C. M. Langley, Department of Psychology, 3210 Tolman Hall, University of California, Berkeley, CA 94720. date. If the simultaneous control of all three factors in the same experiment were to eliminate element superiority, these alternatives would have to be considered the better hypotheses to account for the effect than the limited capacity hypothesis. Alternatively, if element superiority remains, the limited capacity hypothesis would be strongly supported.

The limited capacity hypothesis (Maki \& Leith, 1973; Maki \& Leuin, 1972; Maki et al., 1976) posits a central cognitive attentional channel of limited capacity through which stimulus information must pass in order to be acted on at the time of test. The limited capacity interpretation argues that a pigeon is able to process one element presented as a sample, but that the pigeon becomes overloaded when it must simultaneously process information about two elements of a compound sample. In addition to the element superiority effect, at least three other findings support a limited capacity interpretation. First, matching accuracy for both element and compound samples improves as sample duration increases (Cook et al., 1992; Lamb \& Riley, 1981; Maki \& Leith, 1973; Maki \& Leuin, 1972). That is, the amount of information proessed prior to sample offset increases as sample duration is increased. Second, when sample duration required for matching at a moderate level of performance (approximately $80 \%$ correct) is measured on element and compound trials, compound sample durations are around twice as long as element-sample durations (Maki \& Leith, 1973; Maki \& Leuin, 1972). Third, the element superiority effect can be reduced by making the feature of the compound to be tested predictable, either by using a precue (Lamb, 1988) or by testing for only color or line information over a long series of trials (Leith \& Maki, 1975).

In contrast to the limited capacity account, one alternative hypothesis-generalization decrement-assumes 
that matching performance is the result of generalization of the pecking response from sample to a visually similar test stimulus. Element tests used in a standard DMTS procedure provide a visually identical match to element samples, but not to compound samples. Consequently, generalization decrement might occur when pigeons attempt to match element tests to a compound sample, resulting in low accuracy (Cox \& D'Amato, 1982; Maki et al., 1976; Roberts \& Grant, 1978). According to a second alternative, the coding decrement hypothesis, element superiority may be the result of a frequently used DMTS training procedure in which pigeons are first taught to match element stimuli to a high criterion of performance, and then are transferred to compound stimuli. With this training procedure, birds may develop strong memorial codes representing elements and may later use these element codes ineffectively to represent compound-sample information, resulting in poor matching performance on compound trials (Grant \& MacDonald, 1986). Finally, the gaze direction hypothesis offered by Kraemer, Mazmanian, and Roberts (1987) argues that some compound samples are more difficult to process than elements because the bird must actively shift its gaze from one location to another to acquire information about both elements of the compound. By contrast, it need look at only one location for an element sample. The gaze direction hypothesis places the overload for amount of information that can be encoded at a peripheral level-the number of locations that must be examined-rather than at a central information processing level as suggested by the limited capacity hypothesis.

Past DMTS experiments have failed to decisively test the limited capacity information processing explanation of element and compound matching performance because they have not simultaneously controlled for generalization decrement, coding decrement, and peripheral gaze direction limitations in encoding the samples. In studies in which overlapping line and color elements were used as compounds, experimenters have controlled for gaze direction effects (Cook et al., 1992; Lamb, 1988; Lamb \& Riley, 1981), but the resulting element-compound differences might still be explained by either generalization decrement or coding decrement, since experimental controls for these effects were not included. Conversely, Brown and Morrison (1990) controlled for generalization decrement but used separable compound stimuli, and therefore did not rule out gaze direction effects. Brown and Morrison also addressed the coding decrement interpretation of element superiority. They found that pigeons trained first on compounds showed compound superiority, whereas those trained first on elements showed the usual element superiority. This superiority of matching to the sample type trained first relative to the sample type trained last fits with a coding decrement account. Superior compound matching, however, disappeared for Brown and Morrison's birds trained first on compounds after they had had extensive testing with both sample types, whereas element matching remained superior to compound match- ing for the birds trained first on elements. The fact that both training groups eventually showed element superiority suggests that any coding decrement apparent in early tests no longer influenced matching performance after extensive training, and that coding decrement cannot account for element superiority shown by both groups later in training and testing. This account, however, cannot be dismissed as an explanation of the initial element superiority shown by the birds trained on elements first.

Our goal in the present experiment was to incorporate controls for these three alternative hypotheses and to determine whether an element-compound performance difference would nonetheless remain. First, to control for gaze direction, we used unified compound samples to ensure that the pigeon was exposed to both color and form information while looking in a single location anywhere on the sample. Because the form was made from colored lines, form processing would also result in color processing. In addition, even if effective gaze were restricted to perhaps a single degree of visual angle, the difference between the curvature of a circle or the straight lines and angles of a triangle would still be discernible. Second, we controlled for generalization decrement by using a symbolic matchingto-sample design. Because neither element nor compound samples are physically related to symbolic test stimuli in such a design, any observed element-compound difference could not be attributed to the fact that test stimuli look more like element samples than like compound samples. Third, coding decrement was ruled out by training pigeons simultaneously on element and compound samples, which should result in the development of memorial representations for both types of samples at the same time during training. A manipulation of sample duration was also included in the experiment. Improvement in matching accuracy as sample duration increases has been explicitly predicted by the limited capacity hypothesis, but not by the alternative hypotheses described above.

\section{METHOD}

\section{Subjects}

The subjects were 5 White Carneau pigeons (Columba livia) obtained from a local squab producer. They were housed in individual cages with free access to red grit and water in a colony room on a 14:10-h light:dark cycle. They were maintained at $80 \%-85 \%$ of their individual free feeding weights. The subjects were experimentally naive at the commencement of the experiment.

\section{Apparatus}

The experiment was conducted in two identical three-key operant chambers. Birds 88, 89, and 90 were trained and tested in Apparatus 1; Birds 54 and 55 were trained and tested in Apparatus 2. Each chamber measured $37.7 \mathrm{~cm}$ high, $31.2 \mathrm{~cm}$ deep, and $36.4 \mathrm{~cm}$ across the front and back walls. The chamber walls were painted flat black, with the exception of one Plexiglas sidewall, which served as a door and which was draped with opaque brown felt during the experiment. Three pecking keys (BRS/LVE Model 121-16) on the front wall were $23.4 \mathrm{~cm}$ above the floor. The center key was square $(3.3 \times 3.3 \mathrm{~cm})$, and the side keys were round $(3.0 \mathrm{~cm}$ in diameter). The side keys were separated from the center key by $10.4 \mathrm{~cm}$ edge to edge. The food hopper (BRS/LVE Model 1110) opening, through 
which the subjects received mixed grain reinforcement, was $6.5 \mathrm{~cm}$ square and $15.6 \mathrm{~cm}$ below the center key.

The stimuli were back projected onto the keys by Kodak slide projectors through a series of lenses and beam splitters. The onset and offset of stimuli on the center key was accomplished with highspeed shutters (Uniblitz 214LOAT5H) with rise and fall times of 1.3 and $2.3 \mathrm{msec}$, respectively. The stimuli on the side keys were presented by using Uniblitz Model 26LOA3X5 shutters with a rise time of $1.6 \mathrm{msec}$ and a fall time of $2.6 \mathrm{msec}$. All within-trial events were controlled by a locally constructed microcomputer. All other experimental events were controlled by a Digital Equipment PDP$11 / 23$ computer. Experimental chambers were contained in a windowless room that had been painted flat black.

\section{Stimulus Mapping}

Color-element samples consisted of a $0.6-\mathrm{cm}$ square that was either light blue (B; Kodak Wratten filter No. 38) or green (G; Kodak Wratten filter No. 66), and was centered on the key. Shape elements consisted of a white triangle (T; $1.1-\mathrm{cm}$ base, $1.0 \mathrm{~cm}$ tall) or circle (C: $1.1 \mathrm{~cm}$ in diameter) on a black background. The triangle and circle were not filled figures: they were drawn with lines $0.1 \mathrm{~cm}$ wide. Circles and triangles were also presented in the center of the key. Unified compounds consisted of the triangle or circle figure drawn with blue or green lines; color and shape were coextensive for these spatially unified compound stimuli.

Four different test stimuli were used as symbolic test matches. One pair of test stimuli were thick horizontal lines ( 3 black lines $0.2 \mathrm{~cm}$ wide, each separated by $0.7 \mathrm{~cm}$ of white background) and thin vertical lines ( 6 black lines $0.025 \mathrm{~cm}$ wide, each separated by $0.45 \mathrm{~cm}$ of white background). This pair of test stimuli served as symbolic matches for the shape samples. The symbolic matches for color samples consisted of darkening the top half of one circular side key and the bottom half of the opposite circular side key.

Each bird was presented with test stimuli from one of two sets. For Birds 88,89 , and 90 , the black semicircle on the lower half of the test key was the correct match for the color blue, and the black semicircle on the upper half of the test key was the correct match for green. In addition, a peck to the wide horizontal lines on the test key was correct following a triangle sample, and the thin vertical line stimulus was correct following a circle. For Birds 54 and 55 , these sample-test matches were reversed.

\section{Procedure}

The birds were autoshaped to peck the center key illuminated with white light and were then transferred to the training procedure. They were simultaneously trained on elements and compounds. We determined in a pilot study with the same stimuli (with a different set of birds) that a training procedure showing both element and compound trials each day was unsatisfactory. With this pilot procedure, only 2 of the 3 birds showed performance above chance after over 80 days of training. Given these results, we arrived at the training procedure used in this study, in which the birds were run on elements and compounds on alternate days.

Each trial began with white light on the center key; a peck to this warning signal resulted in the presentation of a sample. During training, 15 pecks to the sample were required. Immediately following the 15th peck, the sample stimulus disappeared and symbolic test stimuli were presented on the side keys. The birds received 320 element or compound trials per day. On compound trial days, 80 trials of each sample appeared (BT, GT, BC, GC). Forty trials for each compound sample were followed by shape tests and 40 by color tests (the dimension tested was unpredictable on any given trial). This number of sample-test stimulus pairings was chosen so that there would be 80 color tests following blue compound stimuli ( $\mathrm{T}$ or $\mathrm{C}$ ) and $\mathbf{8 0}$ color tests following green compound stimuli ( $\mathrm{T}$ or C), and likewise for line tests. On element days, each sample
(B,G,T,C) appeared 80 times. Consequently, there were 80 color tests following the blue-element sample and 80 color tests following the green-element sample. Similarly, there were 80 line tests following both the triangle and the circle samples. Thus, the number of trials on which each sample component $(B, G, T, C)$ was tested was the same on element and compound days.

The position of the correct symbolic test stimulus was counterbalanced across trials from the left to the right side key for both elements and compounds. A peck to the symbolic test stimulus designated correct resulted in access to mixed grain in the illuminated hopper. The length of food reward was adjusted according to the bird's weight: Bird 88 received $1.8 \mathrm{sec}$, and the remaining birds received $1.3 \mathrm{sec}$ of access. Following a peck to the incorrect test stimulus, a 10-sec time-out was given, after which the next trial was presented (i.e., a noncorrection procedure was used). An intertrial interval of $3 \mathrm{sec}$ separated trials. Failure to peck the warning signal within $5 \mathrm{sec}$ or failure to peck one of the test stimuli within $30 \mathrm{sec}$ resulted in a 3-sec pause, followed by re-presentation of the trial. Training was continued until a criterion of $80 \%$ or better performance was reached on both elements and compounds each day over 6 consecutive days (during which element and compound days alternated).

Twenty-eight days of testing followed the completion of training. Elements and compounds were again tested on alternate days. As in training, 320 trials per day were run, but there was no longer a required number of pecks to the sample prior to sample offset. During testing, sample duration was strictly controlled. Each day consisted of 32 warm-up trials with a sample duration of 2185 msec. Test trials followed warm-up and consisted of 48 trials with each of six sample durations: $40,110,295,805,2185$, and $5935 \mathrm{msec}$, for a total of 288 trials. For each sample duration, the following factors were counterbalanced: sample type, location of correct test match on side keys, and dimension tested following compound samples. The order of trial types within days was randomized.

The experiment was run in two phases, the only difference between the two being that in the first phase the colors presented were blue and green (Kodak Wratten, No. 66) and in the second the colors presented were blue and yellow (Kodak Wratten, No. CCY25). Unfortunately, the green color slides (both element and compound) faded during the course of the first phase. Although the overall change in color was imperceptible in daily checks of the apparatus, the color fading was noticed near the end of the first phase. We wanted to rule out the possibility that the results may have been attributable to the gradual color change, so we ran a second 28 days of testing in Phase 2 to validate the results of Phase 1. The more stable yellow wratten filter used in the second phase looked to be about the same color as the green wratten after it had faded. The data from both phases were, however, nearly identical (exceptions are reported below), so the data from first and second phases have been combined for analysis, although the training results are described separately.

\section{RESULTS}

\section{Phase 1 Training}

Table 1 shows the day on which each bird had first run 3 successive days at $80 \%$ accuracy for element days and for compound days. Three birds $(54,55$, and 89$)$ completed their first 3 successive days at $80 \%$ accuracy for elements within 1 day of having completed their first 3 successive days at $80 \%$ accuracy for compounds. Bird 88 completed 3 successive element days at $80 \%$ prior to completing 3 successive compound days at $80 \%$, whereas 
Table 1

Training Day on Which Each Bird Had First Run 3 Successive Days at $\mathbf{8 0} \%$ Accuracy for Element Days and for Compound Days

\begin{tabular}{ccc}
\hline Bird & Element & Compound \\
\hline 54 & 26 & 25 \\
55 & 10 & 11 \\
88 & 50 & 87 \\
89 & 36 & 35 \\
90 & 49 & 27 \\
$M$ & 34.2 & 37.0 \\
\hline
\end{tabular}

Bird 90 did the opposite. The overall results indicate that the birds learned to match to element and to compound samples at about the same rate.

The mean number of days to reach the training criterion ( 6 successive days at $80 \%$ accuracy) for all birds in the first phase was 44 (range, 11-87). A withinsubjects analysis of variance (ANOVA) (sample type $\times$ dimension) was performed on matching accuracy for the final 6 days of training over which the bird met the training criterion. For each bird, accuracy was averaged over the 3 element days, as was accuracy from the 3 compound days, resulting in one datum point per condition per bird. The percentages correct for element and compound trials were $88.8 \%$ and $82.6 \%$, respectively. Elementmatching accuracy was significantly higher than compound matching $[F(1,4)=26.48, p<.01]$, demonstrating that the element superiority effect appears immediately in performance.

\section{Phase 2 Training}

Since the stimuli in the second phase differed only by a slight change in one of the colors from those in the first, the birds, which had participated in the first phase, rapidly reached asymptotic performance. The mean number of days for the birds to reach criterion on the second phase was 6.2 (range, 6-16).

\section{Testing: Phases 1 and 2}

Warm-up trials (the 32 trials of 2185 -msec sample duration run at the beginning of each day) provide an estimate of matching performance to element and compound samples independent of that obtained on the $\mathbf{2 8 8}$ daily trials involving different sample durations. The warm-up trial data for the 56 experimental days were averaged together to obtain one estimate of performance in each condition for each bird. Averaged over birds, the percentages correct for element and compound warm-up trials were 81.7\% and $76.6 \%$, respectively. A within-subjects ANOVA (sample type $\times$ sample dimension) showed that element accuracy was significantly higher than compound accuracy $[F(1,4)=11.03, p<.05]$ for the daily warm-up trials.

Figure 1 shows element- and compound-matching accuracy collapsed over birds as a function of sample duration for trials on which color information and shape information were tested. The unfilled symbols on Figure 1 indicate daily warm-up trial accuracy for elements and compounds on the same test trial types. Element perfor- mance appears higher than compound performance for both warm-up and test trials. Figure 2 shows element performance averaged over all trials and compound performance averaged over all trials, as a function of sample duration for each bird. The birds generally show a superiority of element performance. Although Bird 89 shows a smaller effect than do the other birds, its element-matching accuracy is higher than its compound-matching accuracy at all durations longer than $110 \mathrm{msec}$.

A within-subjects ANOVA (sample type $\times$ sample dimension $\times$ duration) was performed on the test trial data in which sample duration was varied. Fifty-six days of
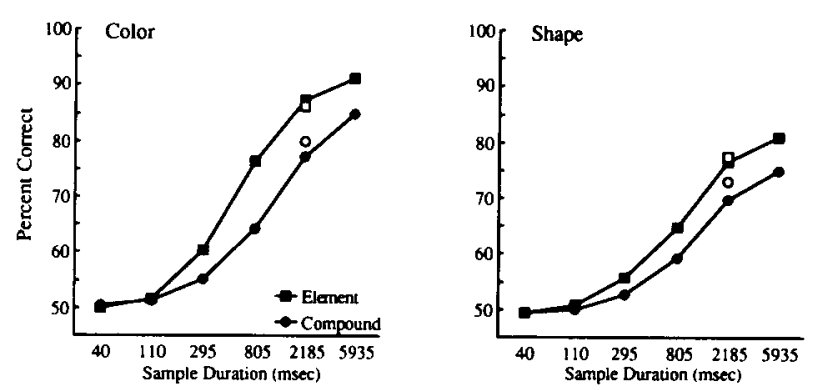

Figure 1. Matching accuracy for color and shape information, as a function of sample duration and sample type. Unfilled circles represent compound accuracy, and unfilled squares represent element accuracy, for daily warm-up trials.
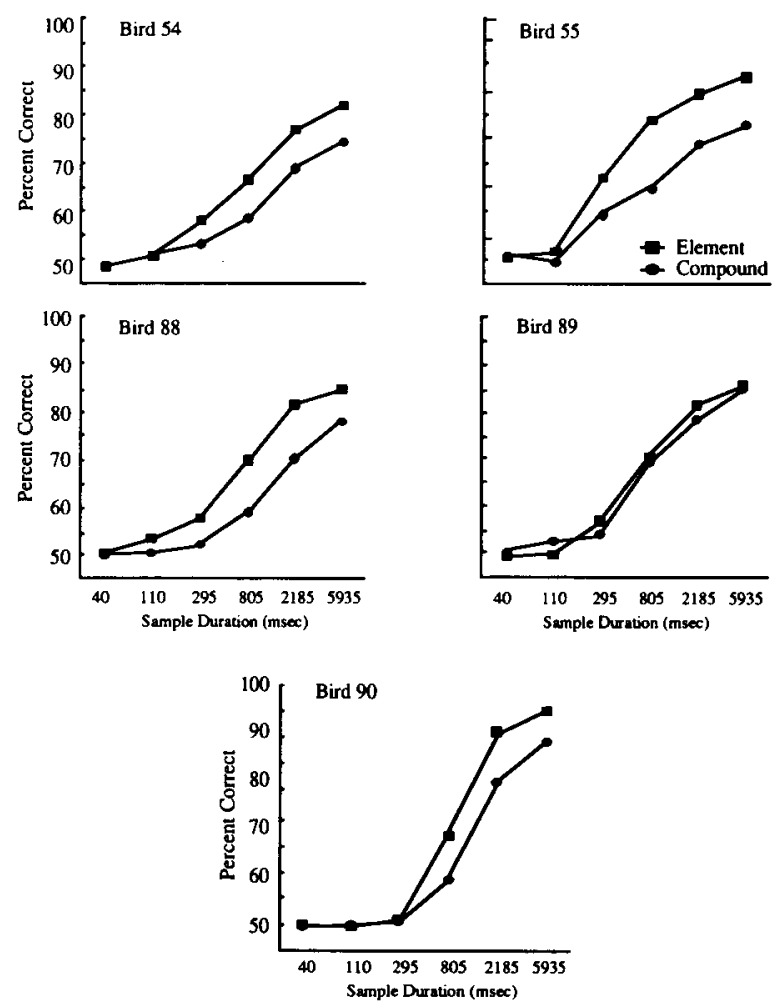

Figure 2. Matching accuracy, as a function of sample duration and sample type for each bird. 
data from each bird were averaged in order to obtain one datum point per condition per bird. Element accuracy was superior to compound accuracy $[F(1,4)=16.35, p<$ $.025]$, and there was a significant improvement in accuracy as sample duration increased $[F(5,20)=124.69$, $p<.001$ ]. Collapsing over both element- and compoundsample trials, matching accuracy was significantly higher when color information was tested than when shape information was tested $[F(1,4)=13.54, p<.05]$.

The sample type $\times$ duration interaction was significant $[F(5,20)=21.86, p<.001]$. Describing the nature of this interaction, trend analyses revealed a significant linear trend of sample duration over element trials $[F(1,4)=$ $559.33, p<.001]$ and over compound trials $[F(1,4)=$ $209.05, p<.001]$. Furthermore, an interaction contrast of the linear components of element and compound samples revealed that the linear trend for compounds differed significantly from that of element trials $[F(1,4)=43.95$, $p<.01]$. This significant interaction of linear trends reflects the increase in the difference between elementand compound-matching accuracy with increasing sample duration.

The sample dimension $\times$ duration interaction was also significant $[F(5,20)=4.68, p<.01]$. Collapsing over element and compound trial data, trend analyses revealed significant linear trends of sample duration for trials on which color information was tested $[F(1,4)=357.13$, $p<.001]$ and for trials on which shape information was tested $[F(1,4)=119.52, p<.001]$. Furthermore, an interaction contrast of the linear components of color trials and shape trials revealed that the linear trend for color accuracy differed significantly from that of shape $[F(1,4)$ $=9.08, p<.05]$. This reflects the increase in the difference between color and shape matching accuracy with increasing sample duration.

All of the statistically significant results reported above (based on the combined data from Phases 1 and 2) were also significant when the data from each phase were analyzed separately, with the following exceptions: Test data from Phase 1 did not show either a main effect of sample dimension $[F(1,4)=2.72, p>.25]$ or an interaction of sample dimension $\times$ duration $[F(5,20)=1.18, p>.10]$.

\section{DISCUSSION}

Superiority of element over compound matching-tosample has been found in a task which simultaneously controls for generalization decrement, coding decrement, and gaze direction, and for any additive or interactive result of two or more of these factors operating together in an experimental design. Simultaneous training of element and compound tasks from the outset of training allowed us to demonstrate that the coding decrement hypothesis could not apply to either early or later element superiority performance: element matching is superior at the end of training and in testing. Similarly, the use of unified compounds and symbolic test stimuli negated any potential influence of gaze direction and generalization decrement, respectively. Finally, the finding of improved performance for elements and compounds as sample duration increases supports a limited capacity interpretation of DMTS performance.

Although these three alternatives to the limited capacity hypothesis were ruled out in the present experiment, two other alternative hypotheses that might account for element superiority remained uncontrolled, however, due in part to the nature of the standard DMTS design. We will refer to these as the test uncertainty hypothesis and the sample-comparison pairing hypotheses.

One consequence of the standard element-compound design is that the test that follows a compound sample is uncertain, whereas the test that follows an element sample is certain. Color tests always follow color-element samples and shape tests always follow shape-element samples, but either a color or a shape test can appear following a compound sample. Test uncertainty following compound samples is included in the design to ensure that the birds must process both color and shape information on each trial in order to match at a high degree of accuracy. It may be the case, however, that the element superiority effect results from test uncertainty per se following compoundsample information encoding, rather than from information overload during the processing of a physically present compound sample. (This interpretation was first suggested to us by E. Wasserman, personal communication).

We have conducted one test of this hypothesis in an unpublished experiment in which we employed only element samples, both color and line, as in previous experiments. In contrast to what was done in previous experimental procedures, each of the four elements had two symbolic test stimuli as correct matches. In test-uncertain blocks of trials, the two symbolic matching test stimuli appeared unpredictably from trial to trial, whereas in test-certain blocks, the symbolic test stimulus for each sample was always the same. Blocks of test-uncertain or test-certain conditions were each run for 5 or 10 days in duration. Matching accuracy in the two conditions was the same, indicating that the element-superiority effect found in element-compound matching-to-sample experiments with the standard design (including the present one) are not attributable to test uncertainty on compound-sample trials.

The same experiment also speaks to the samplecomparison pairing hypothesis. In all previous elementcompound experiments of which we are aware, the frequency of element samples and compound samples has been equated. Because, however, there are two test stimuli for each compound sample but only one for each element sample, the frequency of exposure to each samplestimulus-correct-test-stimulus pairing is half as great in the compound condition as in the element condition. Consequently, the superiority of performance in element conditions could simply be a function of stronger elementsample-test-stimulus associations. We found no difference due to this variable in our test certainty versus uncertainty 
study, even though the frequency of sample-stimulus-teststimulus pairings was twice as great in the test-certain condition as in the test-uncertain condition.

In view of the findings from the present experiment and from the unpublished experiment described above, we conclude that the element superiority effect is the result of a central information processing overload, rather than a difference in element- and compound-sample memory codes or a peripheral sensory limitation. Two remaining challenges to the limited capacity hypothesis seem likely to shape the direction of future research on elementcompound matching-to-sample. The first challenge to the limited capacity hypothesis comes from research showing that some compound stimuli can be matched with the same degree of accuracy as can the elements that make up those compounds. The limited capacity hypothesis proposes that all stimulus information must pass through a single central attentional channel of limited capacity in order to be acted on at the time of test (Maki \& Leith, 1973; Maki et al., 1976). If element superiority does result from a bottleneck in information flow through this single channel, combinations of any types of information should be equally likely to lead to an overload of the channel. Although the present experiment demonstrates a processing decrement for unified color and shape compounds relative to color or shape elements, Kraemer et al. (1987) did not find the element superiority effect when they used unified color and spatial location compounds. Other experiments with separable ambient color/auditory compounds (Kraemer \& Roberts, 1985) or ambient brightness/line compounds also have not found an element superiority effect and, therefore, provide a challenge to the limited capacity hypothesis.

In human cognition research, there is evidence that processing resources may not be shared between some tasks (Allport, 1980; Allport, Antonis, \& Reynolds, 1972; Treisman \& Davies, 1973). Concurrent processing of similar information (two auditory recordings or two visual stimulus lists) may lead to interference, whereas simultaneous processing of dissimilar information (an auditory and a visual stimulus list) can result in performance equivalent to that observed for processing either type of information alone. The pigeon DMTS element-compound research may parallel the human cognition research: Although decrements in simultaneous processing occur for color/line and spatial/spatial (Wilkie \& Summers, 1982) compounds, they do not occur for color/spatial (Kraemer et al., 1987), ambient color/auditory (Kraemer \& Roberts, 1985), or ambient brightness/line compounds (Kraemer \& Roberts, 1987). These results suggest that the failure to find the element superiority effect may occur when elements of a compound are dissimilar and therefore do not compete for central processing resources. For this reason, experiments in which element superiority has not been found do not lead us to reject the notion that central processing interference can occur, but they do lead us to suggest that systematic tests, within and between dimensions, need to be performed so that we can predict which stimulus sets will produce processing overload and which will not.

A second significant challenge for the limited capacity hypothesis is that matching accuracy for element and compound samples does not converge when samples are presented for very long durations, as predicted by the hypothesis. The reasoning is that if enough time were given, all available information from compound samples should be detected and stored by the pigeon, thus yielding convergence of element and compound matching performance. However, the most common finding is that the element-compound difference persists over long sample durations, as it did in the present research. It seems likely, given the persistent failure to find convergence with extended sample durations (Brown \& Morrison, 1990; Cook et al., 1992; Lamb \& Riley, 1981; Roberts \& Grant, 1978; Santi et al., 1982; but see Maki \& Leith, 1973; Maki et al., 1976), that information overload occurs at some stage of processing later than initial information uptake.

That the bottleneck in processing may occur later in the system is suggested by the results of DMTS experiments performed by Lamb (1991). Lamb found an elementcompound difference with human subjects in a standard matching-to-sample task in which reaction times to the test stimuli were the measure (for his subjects, accuracy was at ceiling for both sample types). Specifically, subjects were faster to respond to the test stimuli following element samples as opposed to following compound samples. Lamb also showed that increases in sample duration lead to faster reaction times at test. Lamb's most interesting result, however, was that his subjects showed equally fast times on element and compound trials when he inserted a delay between onset of the test stimuli and the time at which the subject was allowed to respond. Lamb argued that the element-compound difference is due to the difference in time required to retrieve information about the sample from memory: it takes longer to retrieve color information about a compound than about a color element because there are two bits of information (color and shape) in memory following a compound (as opposed to one following an element). Therefore, when subjects are given sufficient time to search memory prior to responding to the color- or line-test comparisons, the difference between element and compound matching disappears.

The present experiments, along with previous investigations (Brown \& Morrison, 1990; Cook et al., 1992; Lamb, 1988; Lamb \& Riley, 1981), lead to the conclusion that the element superiority effect is the result of a central information processing overload during compound trials. The lack of an element superiority effect reported for some types of compounds suggests that future information processing accounts of element-compound matching-tosample performance may have to diverge from the original single processing channel model described by the limited capacity hypothesis. Also, the lack of convergence of element and compound matching at long sample durations suggests that the overload in processing may not oc- 
cur during initial sample information encoding, but elsewhere in the system. Future models must address where in the information processing system information overload may occur. A hypothesis that would provide a complete understanding of information processing in an elementcompound DMTS task should be able to explain the initial findings believed to support the limited capacity hypothesis and more recent findings that have challenged the limited capacity hypothesis in its original form.

\section{REFERENCES}

ALLPORT, D. A. (1980). Attention and performance. In G. Claxton (Ed.), Cognitive psychology: New directions (pp. 112-153). London: Routledge \& Kegan Paul.

Allport, D. A., Antonis, B., \& Reynolds, P. (1972). On the division of attention: A disproof of the single channel hypothesis. Quarterly Journal of Experimental Psychology, 24, 225-235.

Brown, M. F., \& Morrison, S. K. (1990). Element and compound matching-to-sample performance in pigeons: The roles of information load and training history. Journal of Experimental Psychology: Animal Behavior Processes, 16, 185-192.

Cook, R. G. (1980). Retroactive interference in pigeon short-term memory by a reduction in ambient illumination. Journal of Experimental Psychology: Animal Behavior Processes, 6, 326-338.

COOK, R. G., RileY, D. A., \& Brown, M. F. (1992). Spatial and configural factors in compound stimulus processing by pigeons. Animal Learning \& Behavior, 20, 41-55.

Cox, J. K., \& D'Aмato, M. R. (1982). Matching to compound samples by monkeys (Cebus apella): Shared attention or generalization decrement? Journal of Experimental Psychology: Animal Behavior Processes, 8, 209-225.

Grant, D. S., \& MacDonald, S. E. (1986). Matching to element and compound samples in pigeons: The roles of sample coding. Journal of Experimental Psychology: Animal Behavior Processes, 12, 160-171.

Kraemer, P. J., Mazmanian, D. S., \& Roberts, W. A. (1987). Simultaneous processing of visual and spatial stimuli in pigeons. Animal Learning \& Behavior, 15, 417-422.

Kraemer, P. J., \& Roberts, W. A. (1985). Short-term memory for simultaneously presented visual and auditory signals in the pigeon. Journal of Experimental Psychology: Animal Behavior Processes, 11, 137-152.
Kraemer, P. J., \& Roberts, W. A. (1987). Restricted processing of simultaneously presented brightness and pattern stimuli in pigeons. Animal Learning \& Behavior, 15, 15-24.

LAMB, M. R. (1988). Selective attention: Effects of cueing on the processing of different types of compound stimuii. Journal of Experimental Psychology: Animal Behavior Processes, 14, 96-104.

LAMB, M. R. (1991). Attention in humans and animals: Is there capacity limitation at the time of encoding? Journal of Experimental Psychology: Animal Behavior Processes, 17, 45-54.

LAMB, M. R., \& RILEY, D. A. (1981). Effects of element arrangement on the processing of compound stimuli in pigeons (Columba livia). Joumal of Experimental Psychology: Animal Behavior Processes, 7, 45-58.

LeITH, C. R., \& MAKI, W. S., JR. (1975). Attentional shifts during matching-to-sample performance in pigeons. Animal Learning \& Behavior, 3, 85-89.

MAKI, W. S., JR., \& LeITH, C. R. (1973). Shared attention in pigeons. Journal of the Experimental Analysis of Behavior, 19, 345-349.

MAKI, W. S., JR., \& LEUIN, T. C. (1972). Information processing by pigeons. Science, 176, 535-536.

MAKI, W. S., JR., RiLEY, D. A., \& LeITH, C. R. (1976). The role of test stimuli in matching to compound samples by pigeons. Animal Learning \& Behavior, 4, 13-21.

RoberTs, W. A., \& Grant, D. S. (1978). Interaction of sample and comparison stimuli in delayed matching-to-sample with the pigeon. Journal of Experimental Psychology: Animal Behavior Processes, 4 , 68-82.

Santi, A., Grossi, V., \& Gibson, M. (1982). Differences in matchingto-sample performance with element and compound sample stimuli in pigeons. Learning \& Motivation, 13, 240-256.

Treisman, A. M., \& Davies, A. (1973). Divided attention to ear and eye. In S. Kornblum (Ed.), Attention and Performance IV (pp. 101125). New York: Academic Press.

Wilkie, D. M., \& Summers, R. J. (1982). Pigeons' spatial memory: Factors affecting delayed matching of key location. Journal of the Experimental Analysis of Behavior, 37, 45-56.

(Manuscript received June 26, 1991; revision accepted for publication December 18, 1992.) 